MITSUBISHI ELECTRIC RESEARCH LABORATORIES

http://www.merl.com

\title{
Two-Level State Estimation Method for Power Systems with SCADA and PMU Measurements
}

\author{
Sun, H.; Wang, Z.; Nikovski, D. \\ TR2012-028 May 2012
}

\begin{abstract}
This paper proposes a two-level hybrid state estimation method for power systems with SCADA and PMU measurements. A power system is decoupled into PMU observed areas and SCADA observed areas based on its system measurement configuration and topology connectivity, and the states of areas are solved by a two-level computational procedure. The first level uses PMU measurements and pseudo measurements derived from SCADA measurements to formulate a linear state estimation model for each PMU observed area, from which the states of the buses in the area are estimated. The second level uses the SCADA measurements and pseudo measurements generated by results at the first level to formulate a nonlinear state estimation model for each SCADA observed area. The weighted least square method is used to solve the models at the two levels. Test results on an IEEE 14 bus system and an IEEE 118 bus system are given to demonstrate the effectiveness of the proposed method.
\end{abstract}

2012 IEEE PES Innovative Smart Grid Technologies Conference - Asia (ISGT Asia)

This work may not be copied or reproduced in whole or in part for any commercial purpose. Permission to copy in whole or in part without payment of fee is granted for nonprofit educational and research purposes provided that all such whole or partial copies include the following: a notice that such copying is by permission of Mitsubishi Electric Research Laboratories, Inc.; an acknowledgment of the authors and individual contributions to the work; and all applicable portions of the copyright notice. Copying, reproduction, or republishing for any other purpose shall require a license with payment of fee to Mitsubishi Electric Research Laboratories, Inc. All rights reserved. 



\title{
Two-Level State Estimation Method for Power Systems with SCADA and PMU Measurements
}

\author{
Hongbo Sun, Senior Member, IEEE, Zhenhua Wang and Daniel Nikovski, Member, IEEE
}

\begin{abstract}
This paper proposes a two-level hybrid state estimation method for power systems with SCADA and PMU measurements. A power system is decoupled into PMU observed areas and SCADA observed areas based on its system measurement configuration and topology connectivity, and the states of areas are solved by a two-level computational procedure. The first level uses PMU measurements and pseudo measurements derived from SCADA measurements to formulate a linear state estimation model for each PMU observed area, from which the states of the buses in the area are estimated. The second level uses the SCADA measurements and pseudo measurements generated by results at the first level to formulate a nonlinear state estimation model for each SCADA observed area. The weighted least square method is used to solve the models at the two levels. Test results on an IEEE 14 bus system and an IEEE 118 bus system are given to demonstrate the effectiveness of the proposed method.
\end{abstract}

Index Terms-- Least squares methods; Measurement; Phasor Measurement Units; Power Systems; SCADA Systems; State Estimation

\section{INTRODUCTION}

$\mathrm{S}_{\mathrm{P}}$ TATE estimation is one of the critical functions of the power system control center of a power utility company. The state estimator determines the best estimate of the current state of the system, based on the available measurements from various measuring systems. Traditionally, the measurements are collected by supervisory control and data acquisition (SCADA) systems. SCADA systems provide real-time snapshots of power flows and bus voltages, but these snapshots might not be taken simultaneously. Various state estimation methods based on SCADA measurements have been proposed [1], including weighted least square estimator [2]-[3], nonquadratic estimator [4], least absolute value estimator [5], leverage point estimator [6], and least median of squares estimator[7]. Weighted least square estimators are widely accepted in practice. With the increasing adoption of synchronized phasor measurement units (PMU) in power systems, PMU devices are gradually becoming a common data source for the state estimation as well. PMUs provide synchronized measurements across dispersed locations, and furthermore, the measurement frequency and accuracy of PMUs are much higher than those of SCADA systems. Many weighted least square based methods [8]-[11] have

Hongbo Sun is with the Mitsubishi Electric Research Laboratories, Cambridge, MA 02139 USA (e-mail: hongbo.sun@merl.com).

Zhenhua Wang is with the Holcombe Department of Electrical and Computer Engineering, Clemson University, Clemson, SC 29634 USA (email: zhenhuw@clemson.edu).

Daniel Nikovski is with the Mitsubishi Electric Research Laboratories, Cambridge, MA 02139 USA (e-mail: nikovski@merl.com). been proposed to utilize PMU measurements in the state estimation procedure. Some methods used PMU measurements only [8], and others used both SCADA and PMU measurements [9]-[11].

This paper proposes a two-level hybrid state estimation method for power systems with SCADA and PMU measurements. Based on the system measurement configuration and topology connectivity, a power system is decoupled into PMU-observed areas and SCADA-observed areas to be solved by a two-level computational procedure. The first level uses PMU measurements and pseudo measurements derived from SCADA measurements to formulate a linear state estimation model in rectangular coordinates for each PMU observed area, from which the states of buses of the area are estimated. The second level uses the SCADA measurements and pseudo measurements generated by results at the first level to formulate a nonlinear state estimation model in polar coordinates for each SCADA observed area. The weighted least square method is used to solve the models at the two levels. Test results on several IEEE test systems including a 14-bus test case and a 118-bus test case are given to demonstrate the effectiveness of the proposed method. Compared with single level method in which all SCADA and PMU measurements are used simultaneously in the estimation model, the twolevel method reduces the computational time of state estimation significantly, while maintaining almost the same level of estimation accuracy.

\section{THE PROPOSED METHOD}

\section{A. PMU and SCADA Observed Areas}

Based on the location, type and data source of available measurements, a power system is divided into PMUobserved areas and SCADA-observed areas.

For a PMU-observed area, the state of a bus is either directly measured by a PMU, or indirectly determined by the adjacent bus with PMU installation. For a SCADAobserved area, the state of a bus is determined by the SCADA measurements within the area.

State estimation is decoupled into a two-level procedure. The first level is to obtain the state for the PMU-observed areas. The second level is to obtain the states for the SCADA-observed areas.

Fig. 1 shows an example construction of PMU and SCADA observed areas for a power system with PMU and SCADA measurements. In the figure, any single-line circle represents a SCADA-measured bus, and any double-line circle represents a PMU-measured bus. Any single-line branch is SCADA measured, and double-line branches are PMU measured. Buses 1-3 are PMU-measured buses, and 
buses 4-12 are SCADA measured one.
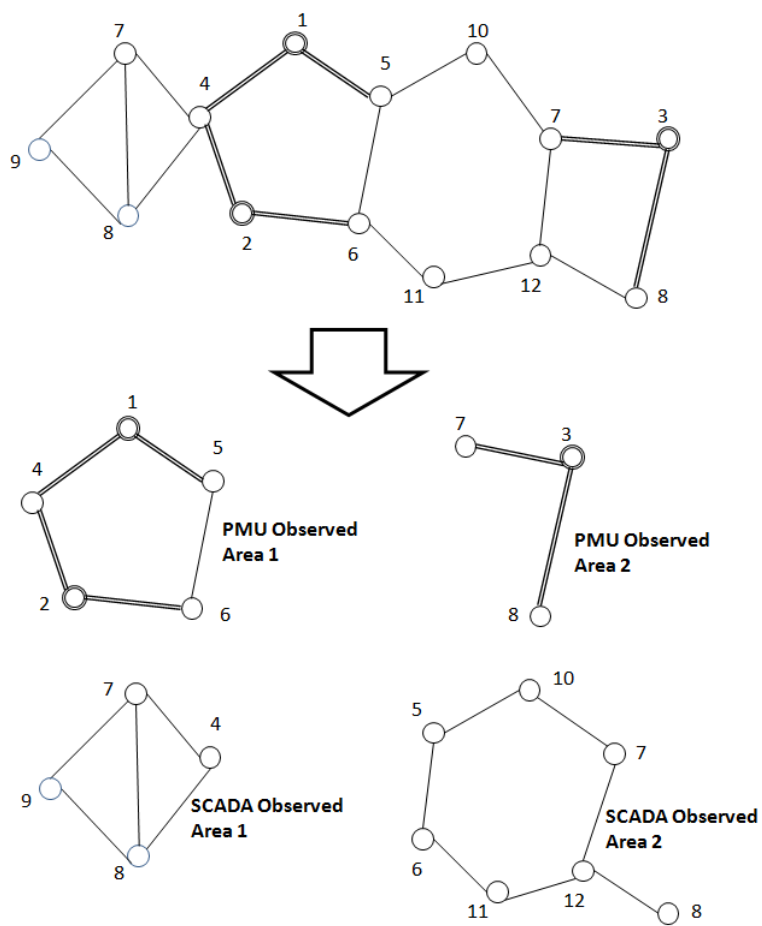

Fig. 1. PMU and SCADA Observed Areas

The system in Fig. 1 is replaced by two PMU-observed areas and two SCADA-observed areas.

PMU observed area 1 consists of buses 1-2 and buses 46 , in which buses 1 and 2 are equipped with PMUs. PMU observed area 2 consists of bus 3 and buses 7-8, in which bus 3 is equipped with PMUs.

SCADA observed area 1 consists of bus 4 and buses 7-9, in which bus 4 is a common bus between PMU area 1 and SCADA area 1. Bus 4's state is determined through a linear estimation model for PMU area 1 first, then taken as pseudo measurements to be resolved with nonlinear estimation model for SCADA area 1. The SCADA observed area 2 consists of buses 5-8 and buses 10-12. Buses 5-7 and bus 12 are boundary buses between the area and PMU area 1 and 2 . Similarly, the states of those buses are solved first in the first level, and then updated in the second level solution.

\section{B. Weighted least square estimation with PMU measurements}

For a PMU-observed area, the relationship between the measurements and state variables can be described as follows:

$$
\mathbf{z}=\mathbf{H} \mathbf{x}+\mathbf{e}
$$

where, $\mathbf{Z}$ is the measurement vector, $\mathbf{x}$ is the state vector, $\mathbf{H}$ is a constant matrix to represent the linear relationship of the measurements to the states, and $\mathbf{e}$ is the measurement error vector and its covariance matrix is $\mathbf{R}, \mathbf{R}=E\left\{\mathbf{e} \bullet \mathbf{e}^{T}\right\}$, where $\mathbf{e}^{T}$ is the transpose of vector $\mathbf{e}$, and $E\{$.$\} stands for expectation. Assuming that the$ distribution of measurements follows a normal distribution, the covariance matrix is a diagonal matrix, and its $i-t h$ diagonal element is the variance of the i-th measurement, $\sigma_{z_{i}}^{2}$.

Rectangular coordinates are used to formulate the state estimation model for PMU-observed areas. The states for each PMU-observed area are represented by the real and imaginary parts of complex bus voltages for each bus in the area.

Measurements are collected from two data sources: one is the group of PMU devices, and the other is the set of pseudo measurements derived from branch power flow measurements of SCADA systems.

The PMU measurements include the real and imaginary parts of complex bus voltages, real and imaginary parts of branch currents for lines or transformers, and real and imaginary parts of bus current injections. It requires all bus voltage and branch current measurement and their standard deviations to be provided in rectangular coordinates. However, those values are commonly collected in polar coordinates, and have to be converted into rectangular form before they are used in the state estimation procedure.

If a required measurement variable is not available, but can be determined from other known actual or pseudo measurement variables, the following equation can be used to determine its standard deviation:

$$
\sigma_{z_{i}}=\sqrt{\sum_{j=1}^{m}\left(\sigma_{y_{j}} \frac{\partial f}{\partial y_{j}}\right)^{2}}
$$

where, $z_{i}$ and $\sigma_{z_{i}}$ are the required variable and its standard deviation, $y_{j}$ and $\sigma_{y_{j}}$ are the $\mathrm{j}$-th associated known variable and its standard deviation that is used to determine the required variable, $f$ is a scalar function to express the relationship between required variable and known variables as shown in (3):

$$
z_{i}=f\left(y_{1}, y_{2}, \ldots, y_{m}\right),
$$

$\partial f / \partial y_{j}$ is the derivative of the required variable with respect to the known variable $y_{j}$, and $\mathrm{m}$ is the total number of associated variables used to determine the required one.

The pseudo measurements to be used are the branch currents calculated from SCADA branch power measurements, and the PMU measured bus voltage at the terminal that SCADA branch powers are measured. Based on the relationship between branch power, bus voltage and branch current, the pseudo branch current measurements can be easily determined. After that equation (2) can be used to determine the standard deviations for the pseudo current measurements accordingly. If the PMU bus voltage at the required terminal is not available, a calculated bus voltage at the terminal has to be determined first, and then use together with SCADA measurements to create the required pseudo branch current measurements. The PMU bus voltage and branch current measured at the opposite terminal of the branch are used to determine the calculated bus voltage and its standard deviation at the required terminal.

$\mathbf{H}$ is constructed based on the relationship between the measurement variables and state variables. The branch current equations and bus current injection equations may be used to determine the values of its matrix elements.

The following normal equation is used to solve for the system states:

$$
\mathbf{G} \mathbf{x}=\mathbf{H}^{T} \mathbf{R}^{-1} \mathbf{z},
$$


where $\mathbf{G}$ is the gain matrix, $\mathbf{G}=\mathbf{H}^{T} \mathbf{R}^{-1} \mathbf{H}$. The sparse Cholesky's decomposition algorithm is used to solve the state estimate expressed in (4).

The covariance of the estimate is $\operatorname{Cov}(\hat{\mathbf{x}})=\mathbf{G}^{-1}$. For simplification purposes, the standard deviation of any state estimate is approximately calculated as follows:

$$
\sigma_{\hat{x}_{i}}=\sqrt{\hat{x}_{i} /\left(\mathbf{H}^{T} \mathbf{R}^{-1} \mathbf{z}\right)_{i}}
$$

where, $\hat{x}_{i}$ is the $\mathrm{i}$-th state estimate, $\sigma_{\hat{x}_{i}}$ is the standard deviation of the estimate, and $\left(\mathbf{H}^{T} \mathbf{R}^{-1} \mathbf{z}\right)_{i}$ is the i-th element of right hand side of (4).

\section{Weighted least square estimation with SCADA measurements}

For a SCADA observed area, the measurements $\mathbf{Z}$ and state variables $\mathbf{X}$ can be related as follows:

$$
\mathbf{z}=\mathbf{h}(\mathbf{x})+\mathbf{e}
$$

where, $\mathbf{h}(\mathbf{x})$ is a nonlinear relationship between the measurement and state variables.

Polar coordinates are used to formulate the state estimation model for the SCADA observed areas.

Measurements for the SCADA areas are mainly coming from the SCADA systems. The measurements to be used include the bus voltage magnitude, the branch active and reactive power flows for a line or transformer, and the bus active and reactive power injections.

The other measurements to be used are coming from the first level estimation results, that is, the estimated voltage magnitude and phase angle at the boundary buses between the area with PMU areas. Equation (5) is used to calculate the standard deviations of those pseudo measurements in rectangular coordinates, and then equation (2) is used to converted them into polar coordinates.

The state variables are the bus voltage magnitudes and the bus phase angles.

An iterative procedure is used for solving (6). The normal equation used for solving for the state changes at each iteration is:

$$
\mathbf{G} \Delta \mathbf{x}=\mathbf{H}^{T} \mathbf{R}^{-1} \Delta \mathbf{z}
$$

where, $\Delta \mathbf{x}$ is the vector of state variable changes, $\Delta \mathbf{z}=\mathbf{z}-\mathbf{h}(\mathbf{x})$ is the vector of mismatches between measurements and true values, $\mathbf{H}=\partial \mathbf{z} / \partial \mathbf{x}$ is the Jacobian matrix that represents the sensitivity of measurement variables with respect to the state variables, and $\mathbf{G}$ is the gain matrix, $\mathbf{G}=\mathbf{H}^{T} \mathbf{R}^{-1} \mathbf{H}$. The branch power flow equations, and bus power injection equations may be used to formulate the Jacobian matrix.

The sparse Cholesky's decomposition algorithm is used to solve for the state changes expressed in (7). The solution of (7) is repeated until the state changes are small enough, or a given maximum iteration number is reached.

\section{NUMERICAL EXAMPLES}

The proposed two-level state estimation has been tested on several IEEE test systems including a 14 bus system, and a 118 bus system.

In order to demonstrate the effectiveness of the proposed method, both the proposed two-level method and a conventional single-level method are implemented and tested against the IEEE systems.

The state variables of the single-level state estimation method are the voltage magnitudes and phase angles of all buses in the systems. The measurement variables includes all PMU and SCADA measurements, including PMUmeasured bus voltage magnitudes and phase angles, SCADA-measured bus voltage magnitudes, SCADAmeasured bus active and reactive power injections, SCADA-measured branch active and reactive power flows, and the real and imaginary parts of PMU-measured branch currents.

For each test system, 100 measurement samples have been created by means of a Monte-Carlo simulation method. The normal distribution is used to model the stochastic distribution of measurement noise, and the used standard deviations for all measurement types are listed in Table I.

\begin{tabular}{|c|c|c|c|}
\hline & & PMU & SCADA \\
\hline \multirow[t]{2}{*}{ Bus Voltage } & Magnitude(Per Unit) & 0.0002 & 0.003 \\
\hline & Phase Angle(Degrees) & 0.01 & 1 \\
\hline \multirow{2}{*}{$\begin{array}{l}\text { Branch } \\
\text { Current }\end{array}$} & Magnitude(Per Unit) & 0.0003 & 1 \\
\hline & Phase Angle(Degrees) & 0.015 & 1 \\
\hline \multirow{2}{*}{$\begin{array}{c}\text { Branch } \\
\text { Power Flow }\end{array}$} & Active Power(Per Unit) & I & 0.003 \\
\hline & Reactive Power(Per Unit) & 1 & 0.003 \\
\hline \multirow{2}{*}{$\begin{array}{c}\text { Bus Power } \\
\text { Injection }\end{array}$} & Active Power(Per Unit) & 1 & 0.003 \\
\hline & Reactive Power(Per Unit) & / & 0.003 \\
\hline
\end{tabular}

TABLE I

STANDARD DEVIATIONS FOR MEASUREMENTS

\section{A. State Estimation of IEEE 14 Bus System}

Fig. 2 gives the schematic diagram of IEEE 14 Bus system. Its measuring points and measuring types are listed in Table II. For each branch, its measurements can be provided at two different directions, the positive direction and negative direction. The branch measurements at the positive direction are measured at the first terminal bus of two-terminal pair of the branch, and the negative direction at the second terminal bus.

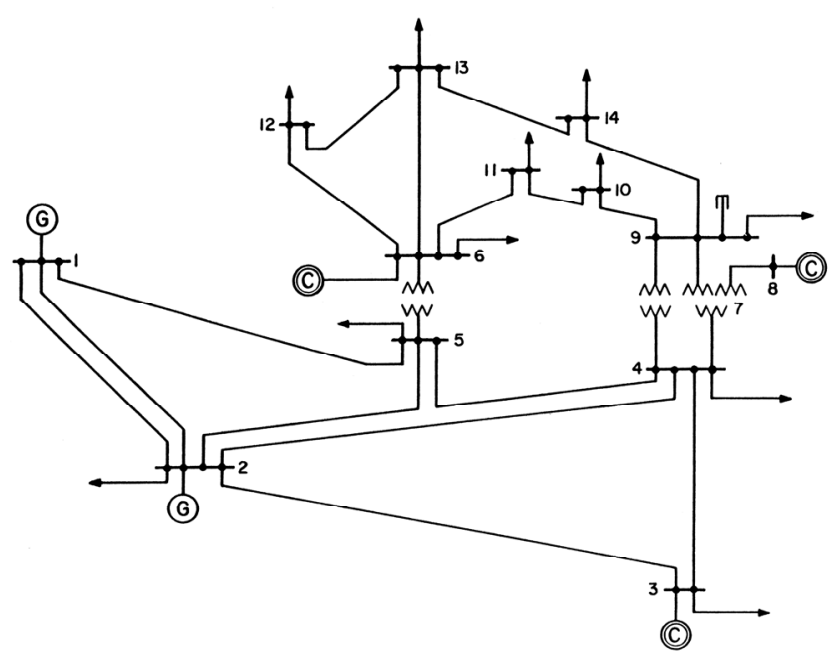

Fig. 2. IEEE 14 Bus Test System

According to the measurement configuration and system topology, the system is divided into PMU and SCADA observed areas. Each area contains several buses and all lines or transformers between those buses. 
TABLE II

MEASUREMENT CONFIGURATION OF IEEE 14 BUS SYSTEM

\begin{tabular}{|c|c|c|c|}
\hline & & PMU & SCADA \\
\hline \multirow{2}{*}{$\begin{array}{c}\text { Bus } \\
\text { Voltage }\end{array}$} & Magnitude & \multirow[t]{2}{*}{$1,6,9$} & $\begin{array}{c}2-5,7-8 \\
10-14\end{array}$ \\
\hline & $\begin{array}{l}\text { Phase } \\
\text { Angle }\end{array}$ & & 1 \\
\hline \multirow{2}{*}{$\begin{array}{c}\text { Branch } \\
\text { Current } \\
\text { (Magnitude } \\
\text { \& Phase } \\
\text { Angle) }\end{array}$} & $\begin{array}{l}\text { Positive } \\
\text { Direction }\end{array}$ & $\begin{array}{c}1 / 2,1 / 5,6 / 11 \\
6 / 12,6 / 13, \\
9 / 10,9 / 14\end{array}$ & \multirow[t]{2}{*}{ / } \\
\hline & $\begin{array}{l}\text { Negative } \\
\text { Direction }\end{array}$ & $5 / 6,4 / 9,7 / 9$ & \\
\hline \multirow{2}{*}{$\begin{array}{l}\text { Branch } \\
\text { Power } \\
\text { (Active \& } \\
\text { Reactive) }\end{array}$} & $\begin{array}{l}\text { Positive } \\
\text { Direction }\end{array}$ & \multirow[t]{2}{*}{ / } & $\begin{array}{c}2 / 3,2 / 4,2 / 5,3 / 4, \\
4 / 5,4 / 7,4 / 9,5 / 6, \\
10 / 11,12 / 13 \\
13 / 14\end{array}$ \\
\hline & $\begin{array}{l}\text { Negative } \\
\text { Direction }\end{array}$ & & $\begin{array}{c}1 / 2,1 / 5,2 / 3, \\
2 / 4,2 / 5,3 / 4, \\
4 / 5,6 / 11,6 / 12 \\
6 / 13,7 / 8,9 / 10 \\
9 / 14,10 / 11 \\
12 / 13,13 / 14\end{array}$ \\
\hline
\end{tabular}

As shown in the table, the 14 bus system has three PMU measured buses, bus 1 , bus 6 and bus 9 . It is partitioned into two areas, one PMU observed area which contains buses 12,4-7, and 9-14, and one SCADA observed area which contains buses 2-5 and 7-8.

Table III gives the computation results of single-level and two-level methods.

TABLE III

COMPARISON OF COMPUTATION PERFORMANCES ON IEEE 14 BUS SySteM

\begin{tabular}{l|c|c|c|c|c}
\hline \multirow{2}{*}{ Method } & \multicolumn{2}{|c|}{$\begin{array}{c}\text { Maximum } \\
\text { Mismatches }\end{array}$} & \multicolumn{2}{|c|}{$\begin{array}{c}\text { Average } \\
\text { Mismatches }\end{array}$} & \multirow{2}{*}{$\begin{array}{c}\text { CPU } \\
\text { Time } \\
\text { (Sec.) }\end{array}$} \\
\cline { 2 - 5 } & $\begin{array}{c}\text { Voltage } \\
\text { (Per } \\
\text { Unit) }\end{array}$ & $\begin{array}{c}\text { Phase } \\
\text { Angle } \\
\text { (Deg.) }\end{array}$ & $\begin{array}{c}\text { Voltage } \\
\text { (Per } \\
\text { Unit) }\end{array}$ & $\begin{array}{c}\text { Phase } \\
\text { Angle } \\
\text { (Deg.) }\end{array}$ & \\
\hline $\begin{array}{l}\text { Single- } \\
\text { level } \\
\text { method }\end{array}$ & 0.00142 & 0.08773 & 0.00015 & 0.00842 & 0.019 \\
\hline $\begin{array}{l}\text { Two- } \\
\text { level } \\
\text { method }\end{array}$ & 0.00555 & 0.11386 & 0.00237 & 0.03480 & 0.003 \\
\hline
\end{tabular}

The accuracy of two-level method is slightly lower than the single-level method. If we look at the maximum estimation error, the maximum errors of the single-level method are 0.00142 per unit in magnitude, and 0.08773 degrees in phase angle. In comparison, the maximum errors of two-level method are 0.00555 per unit in magnitude, and 0.11386 degrees in phase angle.

But the slight sacrifice of estimation accuracy is well rewarded by the gains in computation efficiency. As shown in the table, the computation time for two-level method was 0.003 seconds compared to 0.019 seconds for single-level method.

\section{B. State Estimation of IEEE 118 Bus System}

Fig. 3 is a schematic diagram of the IEEE 118 Bus system. Its measuring points and measuring types are listed in Table IV.

Based on the measurement configuration, the system is divided into three areas: one PMU-observed area which contains buses $24,65,68-71,74-85,96-99$ and 116 , and two SCADA-observed areas, such that the first SCADA area contains buses 1-10,11-67, 69,71-76, 113-115,117, and 118, and the second SCADA area contains buses 82, and 84-112.

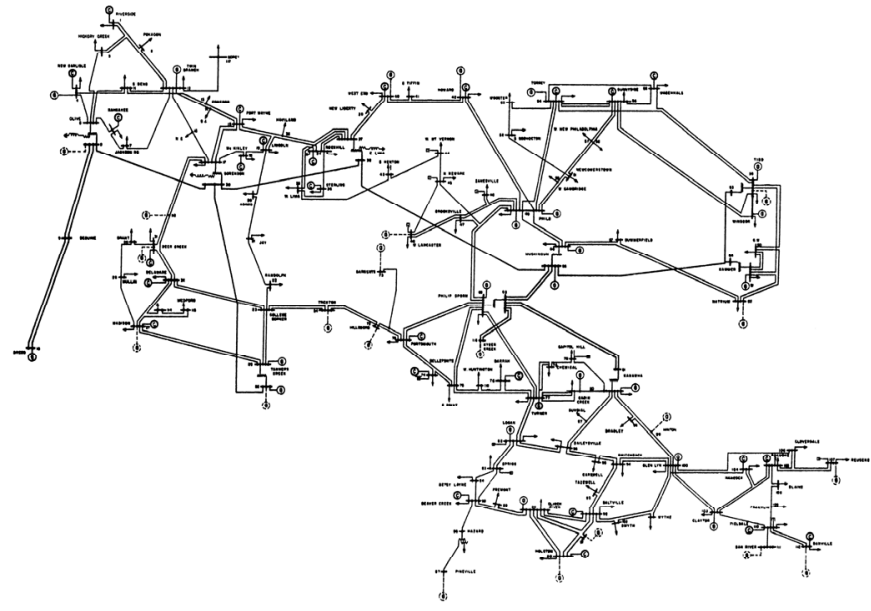

Fig. 3. IEEE 118 Bus Test System

TABLE IV

MEASUREMENT CONFIGURATION OF IEEE 118 BUS SYSTEM

\begin{tabular}{|c|c|c|c|}
\hline & & PMU & SCADA \\
\hline \multirow[t]{2}{*}{$\begin{array}{c}\text { Bus } \\
\text { Voltage }\end{array}$} & Magnitude & \multirow[t]{2}{*}{$\begin{array}{c}68,70,77 \\
80,83\end{array}$} & $\begin{array}{c}1-67,69,71-76 \\
78,79,81,82 \\
84-118\end{array}$ \\
\hline & $\begin{array}{l}\text { Phase } \\
\text { Angle }\end{array}$ & & 1 \\
\hline \multirow{2}{*}{$\begin{array}{c}\text { Branch } \\
\text { Current } \\
\text { (Magnitude } \\
\text { \& Phase } \\
\text { Angle) }\end{array}$} & $\begin{array}{l}\text { Positive } \\
\text { Direction }\end{array}$ & $\begin{array}{l}\text { 70/71,70/74, } \\
70 / 75,77 / 78, \\
77 / 80,77 / 80, \\
68 / 81,77 / 82, \\
83 / 84,83 / 85, \\
80 / 96,80 / 97, \\
80 / 98,80 / 99, \\
68 / 116,68 / 69\end{array}$ & \multirow[t]{2}{*}{ / } \\
\hline & $\begin{array}{l}\text { Negative } \\
\text { Direction }\end{array}$ & $\begin{array}{c}65 / 68,69 / 70, \\
24 / 70,76 / 77, \\
69 / 77,75 / 77, \\
77 / 80,77 / 80, \\
82 / 83 \\
\end{array}$ & \\
\hline \multirow{2}{*}{$\begin{array}{l}\text { Branch } \\
\text { Power } \\
\text { (Active \& } \\
\text { Reactive) }\end{array}$} & $\begin{array}{l}\text { Positive } \\
\text { Direction }\end{array}$ & \multirow[t]{2}{*}{ 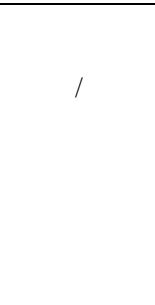 } & \multirow{2}{*}{$\begin{array}{c}\text { 8/5,26/25,30/17, } \\
\text { 38/37,63/59, } \\
\text { 64/61,65/66, } \\
\text { 81/80, and all line } \\
\text { branches except } \\
\text { branches with } \\
\text { PMU branch } \\
\text { current } \\
\text { measurements }\end{array}$} \\
\hline & $\begin{array}{l}\text { Negative } \\
\text { Direction }\end{array}$ & & \\
\hline
\end{tabular}

Table V shows computation results on the single-level and two-level methods of the 118-bus system.

TABLE V

COMPARISON OF COMPUTATION

PERformanCES ON IEEE 118 BUS System

\begin{tabular}{|c|c|c|c|c|c|}
\hline \multirow[t]{2}{*}{ Method } & \multicolumn{2}{|c|}{$\begin{array}{l}\text { Maximum } \\
\text { Mismatches }\end{array}$} & \multicolumn{2}{|c|}{$\begin{array}{c}\text { Average } \\
\text { Mismatches }\end{array}$} & \multirow{2}{*}{$\begin{array}{c}\text { CPU } \\
\text { Time } \\
\text { (Sec.) }\end{array}$} \\
\hline & $\begin{array}{c}\text { Voltage } \\
\text { (Per } \\
\text { Unit) }\end{array}$ & $\begin{array}{l}\text { Phase } \\
\text { Angle } \\
\text { (Deg.) }\end{array}$ & $\begin{array}{c}\text { Voltage } \\
\text { (Per } \\
\text { Unit) }\end{array}$ & $\begin{array}{l}\text { Phase } \\
\text { Angle } \\
\text { (Deg.) }\end{array}$ & \\
\hline $\begin{array}{l}\text { Single- } \\
\text { level } \\
\text { method }\end{array}$ & 0.00134 & 0.10779 & 0.00016 & 0.01850 & 30.434 \\
\hline $\begin{array}{l}\text { Two- } \\
\text { level } \\
\text { method }\end{array}$ & 0.00243 & 0.12362 & 0.00035 & 0.02167 & 4.819 \\
\hline
\end{tabular}

The maximum errors of the single-level method are 0.00134 per unit in magnitude, and 0.10779 degrees in phase angle. The maximum errors of the two-level method are 0.00243 per unit in magnitude, and 0.12362 degrees in 
phase angle. It took 30.434 seconds for the single-level method to find the solution, and 4.819 seconds for the twolevel method.

Compared with single-level method, the two-level method has reduced the computational time of state estimation significantly, while maintaining almost the same level of estimation accuracy.

\section{CONCLUSIONS}

A two-level hybrid state estimation method is proposed for power systems with SCADA and PMU measurements. Based on the system measurement configuration and topology connectivity, a power system is decoupled into PMU observed areas and SCADA observed areas to be solved. The first level uses PMU measurements and pseudo measurements derived from SCADA power flow measurements to formulate a liner state estimation model for each PMU observed area, and the states of buses of the area are determined. The second level uses the SCADA measurements and pseudo measurements generated by results at first level to formulate an nonlinear state estimation model for each SCADA observed area. The weighted least square method is used to solve two level models.

The test results of several IEEE test systems have demonstrated the effectiveness of the proposed method. Compared with single-level method in which all SCADA and PMU measurements are used simultaneously in the estimation model, the two-level method reduces the computational time of state estimation significantly, while maintaining almost the same level of estimation accuracy.

\section{REFERENCES}

[1] A. Monticelli," Electric Power System State Estimation", Proceeding of the IEEE, vol. 88, no.2, pp.262-282, February 2000.

[2] F. C. Schweppe and E. J. Handschin, "Static state estimation in electric power systems", Proceedings of the IEEE, vol. 62, no.7, pp. 972-982, July 1974.

[3] F. C. Schweppe, J.Wildes, and D. Rom, "Power system static state estimation: Parts I, II, and III," IEEE Transactions on Power Apparatus and Systems, vol. PAS-89, no.1, pp. 120-135, January 1970.

[4] H. M. Merril and F. C. Schweppe, "Bad data suppression in power system state estimation", IEEE Transactions on Power Apparatus and Systems, vol. PAS-90, no.6, pp. 2718-2725, November/December 1971.

[5] M. R. Irving, R. C. Owen, and M. Sterling, "Power system state estimation using linear programming," Proc. Inst Elect.Eng., vol. 125 , no.9, pp. 978-885, September 1978.

[6] L. Mili, M. G. Cheniae, N. S. Vichare, and P. J. Rousseeuw, "Robust state estimation based on projection statistics", IEEE Transactions on Power Systems, vol. 11, no.2, pp. 1118-1127, May 1996.

[7] L. Mili, V. Phaniraj, and P. J. Rousseeuw, "Least median of squares estimation in power-systems", IEEE Transactions on Power Systems, vol. 6, no.2, pp. 511-523, May 1991.

[8] A.G. Phadke, J.S. Thorp, and K.J. Karimi, "State Estimation with Phasor Measurements", IEEE Transactions on Power Systems, vol. 1, no.1, pp. 233-241, February 1986.

[9] A.G. Phadke, J.S. Thorp and K.J. Karimi, " Real Time Voltage Phasor Measurements for Static State Estimation", IEEE Transactions on Power Apparatus and Systems, vol. PAS-104, no.11, pp.3098-3107, November 1985.

[10] R. Avila-Rosales, M.J. Rice, J. Giri, L. Beard, and F. Galvan, "Recent experience with a hybrid SCADA/PMU on-line state estimator", in Proc. 2009 IEEE Power \& Energy Society General Meeting, pp.1-8.

[11] S. Chakrabarti, E. Kyriakides, G. Ledwich, and A. Ghosh, "Inclusion of PMU current phasor measurements in a power system state estimator", IET Generation, Transmission, and Distribution, vol. 4, no.10, pp. 1104-1115, September. 2010.

\section{BIOGRAPHIES}

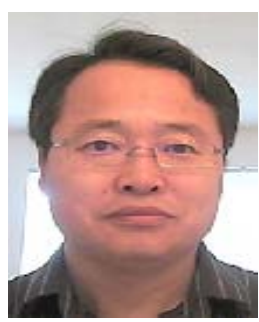

Hongbo Sun(SM' 2000) was born in Liaoning, China in 1966. He received the B.S. degree in Electrical Engineering from Southwest Jiaotong University, China in 1986, and the M.S. and Ph.D. degrees in Electrical Engineering from Chongqing University, China in 1988 and 1991 respectively. $\mathrm{He}$ is a senior member of IEEE, and a registered professional engineer. He is currently working at Mitsubishi Electric Research Laboratories in Cambridge, Massachusetts, USA. His research interests include power system planning and analysis, power operation and control, and smart grid applications.

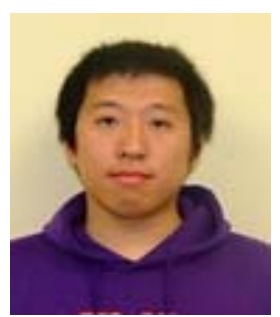

Zhenhua Wang was born in Shanxi, China in 1981. He received BS degree in Electrical Engineering from Zhengzhou University, China in 2003 and MS degree in Electrical Engineering from Tianjin University, China in 2007 respectively. Now he is pursuing the Ph.D. degree at the Holcombe department of Electrical and Computer Engineering, Clemson University, USA. His research interest includes power system transient stability, power system state estimation and wind energypenetration.

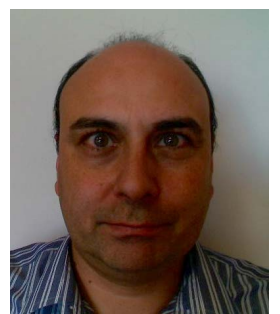

Daniel Nikovski(M' 2003) was born in Plovdiv, Bulgaria, in 1969. He received a B.Sc. degree in Computer Systems and Control from the Technical University Sofia, Bulgaria, in 1992, a M.Sc. degree in Computer Science from the Southern Illinois University in Cabondale, USA, in 1995, and a Ph.D. degree in Robotics from Carnegie Mellon University in Pittsburgh, USA, in 2002. He is currently Senior Member of Research Staff and Group Manager of the Data Analytics group at Mitsubishi Electric Research Laboratories in Cambridge, Massachusetts, USA. His research interests include machine learning, optimization and control, and numerical methods for analysis of complex industrial systems. 